\section{(6) OPEN ACCESS}

\title{
Pan PPAR agonist IVA337 is effective in prevention and treatment of experimental skin fibrosis
}

\author{
Nadira Ruzehaji, ${ }^{1,2}$ Camelia Frantz, ${ }^{1}$ Matthieu Ponsoye, ${ }^{1}$ Jerome Avouac, ${ }^{1,2,3}$ \\ Sonia Pezet, ${ }^{1}$ Thomas Guilbert, ${ }^{1,2,3}$ Jean-Michel Luccarini, ${ }^{4}$ Pierre Broqua, ${ }^{4}$ \\ Jean-Louis Junien, ${ }^{4}$ Yannick Allanore ${ }^{1,2,3}$
}

\begin{abstract}
Handling editor Tore K Kvien
- Additional material is published online only. To view please visit the journal online (http://dx.doi.org/10.1136/ annrheumdis-2015-208029).

${ }^{1}$ INSERM, U1016, Institut Cochin, Paris, France ${ }^{2}$ CNRS, UMR8104, Paris, France

${ }^{3}$ Université Paris Descartes, Sorbonne Paris Cité, Paris, France

${ }^{4}$ Inventiva, Daix, France
\end{abstract}

Correspondence to Dr Nadira Ruzehaji, Institut Cochin, INSERM U1016, Bâtiment Gustave Roussy, 27 rue du Faubourg Saint Jacques Paris, 75014, France; nadira.ruzehaji@inserm.fr

Received 4 June 2015 Revised 3 February 2016 Accepted 21 February 2016 Published Online First 9 March 2016
CrossMark

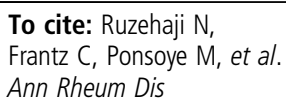

\section{ABSTRACT}

Background The pathogenesis of systemic sclerosis (SSc) involves a distinctive triad of autoimmune, vascular and inflammatory alterations resulting in fibrosis.

Evidence suggests that peroxisome proliferator-activated receptors (PPARs) play an important role in SSc-related fibrosis and their agonists may become effective therapeutic targets.

Objective To determine the expression of PPARs in human fibrotic skin and investigate the effects of IVA337, a pan PPAR agonist, in in vitro and in vivo models of fibrosis.

Methods The antifibrotic effects of IVA337 were studied using a bleomycin-induced mouse model of dermal fibrosis. The in vivo effect of IVA337 on wound closure and inflammation were studied using an excisional model of wound healing.

Results Low levels of PPAR $\alpha$ and PPAR $\gamma$ were detected in the skin of patients with SSc compared with controls. In mice, IVA337 was associated with decreased extracellular matrix (ECM) deposition and reduced expression of phosphorylated SMAD2/3-intracellular effector of transforming growth factor (TGF)- $\beta 1$. Although the antifibrotic effect of pan PPAR was similar to that of PPAR $\gamma$ agonist alone, a significant downregulation of several markers of inflammation was associated with IVA337. Despite its anti-inflammatory and antifibrotic properties, IVA337 did not interfere with wound closure. In vitro effects of IVA337 included attenuation of transcription of ECM genes and alteration of canonical and non-canonical TGF- $\beta$ signalling pathways.

Conclusions These findings indicate that simultaneous activation of all three PPAR isoforms exerts a dampening effect on inflammation and fibrosis, making IVA337 a potentially effective therapeutic candidate in the treatment of fibrotic diseases including SSc.

\section{INTRODUCTION}

Fibrosis is a feature of one of the most debilitating rheumatic diseases-systemic sclerosis (SSc). ${ }^{12}$ The identification of key factors that drive fibrosis are of interest for clinical therapy because to date, no drug has been approved as an antifibrotic capable of preventing progression or reverting existing fibrosis. Pathological fibrogenesis is a complex process activated in response to tissue injury ${ }^{3}$ initiated by endothelial cell apoptosis, which sets off inflammation. ${ }^{4}$ Both the innate and adaptive immune systems are believed to contribute to pathogenesis of fibrosis as the recruitment of immune cells leads to activation of fibroblasts and conversion of latent transforming growth factor (TGF)- $\beta$ into the active form. ${ }^{5}$

Nuclear receptors are a family of transcription factors with key roles in fibrotic responses. ${ }^{6}$ Peroxisome proliferator-activated receptors (PPARs) are nuclear receptors known to modulate fibrosis. ${ }^{7-9}$ PPAR $\alpha$ activator fenofibrate prevented lung fibrosis, ${ }^{10}$ whereas GW0742, a high-affinity PPAR $\delta$ agonist, reduced bleomycin-induced inflammation. ${ }^{11}$ PPAR $\gamma$ agonists including rosiglitazone and pioglitazone attenuated skin and lung fibrosis. ${ }^{9} 12$ The current interest in targeting PPARs for the treatment of fibrosis is based on their ability to modulate gene expression $^{7}$ and inhibit innate inflammation. ${ }^{9} \quad 13$ Given the antifibrotic and anti-inflammatory effects of PPARs, the use of agonists for all three PPARs is expected to lead to greater improvement in efficacy compared with targeting a single PPAR isoform. Furthermore, pan PPAR agonists are expected to offset side effects such as weight gain and bone fractures observed with more selective PPAR agonists. ${ }^{13}$ Thus, a novel pan PPAR agonist-IVA337 was developed, which showed moderate agonist activity for PPAR isoforms. The use of agents with lower affinity (as is the case with IVA337) might be a more suitable therapeutic approach compared with high-affinity PPAR agonists, ${ }^{13}$ which showed some safety concerns. ${ }^{13}$ We herein hypothesised that pan PPAR activation with IVA337 will synergistically reduce profibrotic responses. In the present study, we highlighted the importance of PPARs in fibrogenesis and identified IVA337 as a therapeutic candidate for the treatment of clinically challenging SSc.

\section{MATERIALS AND METHODS}

\section{Human skin collection}

Patients with limited and diffuse SSc, as defined by LeRoy et al, ${ }^{15}$ were used to collect fibrotic skin. ${ }^{16}$ Patients were free from drugs that interfere with dermal matrix formation including steroids and immunosuppressive medications. Informed consent was obtained from patients $(n=15)$ and normal controls $(n=6)$. The study protocol conformed to the ethical guidelines of the 1975 Declaration of Helsinki as reflected in approval by relevant Human Research Ethics Committee.

\section{Animal studies}

Male, aged 6 weeks, C57BL/6 mice (Janvier, Genest-St-Isle, France) were used in three different 
animal trials. (i) Experimental dermal fibrosis (preventative model) ${ }^{16}$ was induced with bleomycin ( $n=6$ each group). Concurrent treatment with local injections of bleomycin $(0.5 \mathrm{mg} / \mathrm{mL})$ and either IVA337 (30 mg/kg), IVA337 (100 mg/kg), rosiglitazone (5 mg/ $\mathrm{kg}$ ) or vehicle by daily oral gavage continued for 3 weeks. Subcutaneous injections of $0.9 \% \mathrm{NaCl}$ served as a control. (ii) Experimental dermal fibrosis (curative model) ${ }^{17}$ was induced using subcutaneous bleomycin for 6 weeks, but 3 weeks after the first injection, mice were given a daily dose of either IVA337 (30 mg/kg), IVA337 (100 mg/kg), rosiglitazone $(5 \mathrm{mg} / \mathrm{kg})$ or vehicle by oral gavage for the remaining 3 weeks. (iii) To investigate the effect of PPARs in physiological tissue repair and excisional model of wound healing ${ }^{18}$ (see online supplementary file). All animal experiments were approved by the relevant Animal Ethics Committee (Comité National de Réflexion Ethique sur l'Expérimentation Animale-34).

\section{Histology and immunohistochemistry}

Histological sections $(4 \mu \mathrm{m})$ were cut from paraffin-embedded formalin-fixed skin tissue. Sections were stained with H\&E and histological dermal thickness was determined by measuring the distance between the epidermal-dermal junction and dermalsubcutaneous fat junction. ${ }^{16}{ }^{19}$ Skin sections were subjected to immunohistochemistry using Leica Bond III (Leica Biosystems, Nanterre, France). Immuno-positive cells were counted ${ }^{16}$ and expressed as a number of total cells within each microscopic filed normalised against $\mathrm{NaCl}$ control. $^{16}{ }^{20}$ Images were captured using a Zeiss AxioObserver.Z1 Microscope (Oberkochen, Germany).

\section{Collagen assessment}

Collagen was visualised using Masson's trichrome staining and second harmonic generation (SHG) imaging (see online supplementary file). ${ }^{21}$ The total collagen content of tissue samples was determined by hydroxyproline assays.

\section{Immunofluorescence staining of skin and fibroblasts}

Sections were deparaffinised in two changes of xylene and placed into $250 \mathrm{~mL}$ target retrieval solution. ${ }^{22}$ Primary antibodies were applied and incubated overnight at $4^{\circ} \mathrm{C}$ (see online supplementary file). Detection was by species-specific secondary antibodies. Image J was used to measure the number of positive cells per unit area. ${ }^{22}$ Immunofluorescence staining of cultured fibroblasts was performed as described previously. ${ }^{23}$ Samples were incubated with primary antibody followed by the appropriate fluorophore-tagged secondary antibody.

\section{STATISTICAL ANALYSIS}

All data were expressed as mean \pm SEM. Statistical analysis was performed using GraphPad Prism 6.04 software (San Diego, California). For a two-group comparison, a Student's $t$ test was used, provided the pretest for normality (D'Agostino-Pearson normality test) was not rejected at the 0.05 significance level; otherwise, a non-parametric Mann-Whitney U test was used.
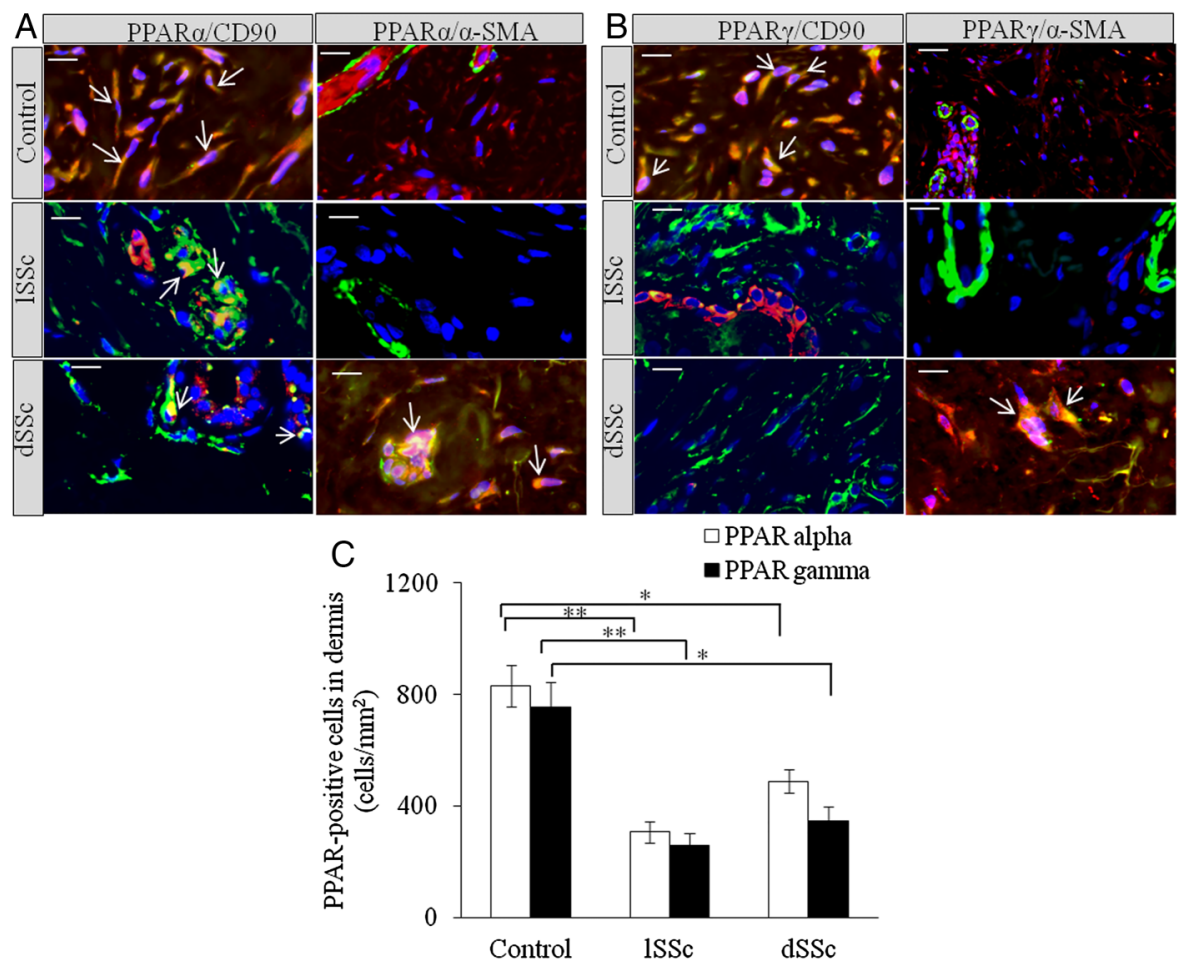

Figure 1 Increased expression of peroxisome proliferator-activated receptor (PPAR) $\alpha$ and PPAR $\gamma$ in human systemic sclerosis (SSc) skin. (A) Representative images of formalin-fixed and paraffin-embedded samples of human healthy control, limited SSc (ISSC) and diffused (dSSc) skin stained with anti-PPAR $\alpha$ (red), anti-CD90 (green) and $\alpha$-smooth muscle actin (SMA) (green) antibodies. (B) Human healthy control, ISSc and dSSc skin stained with anti-PPAR $\gamma$ (red), anti-CD90 (green) and $\alpha$-SMA (green) antibodies. In all images 4',6-diamidino-2-phenylindole (DAPI) (blue) was used for nuclear counterstaining. Images were captured using 100x objective lens. Scale bar is $20 \mu \mathrm{m}$. In normal skin, PPAR $\alpha$ and PPAR $\gamma$ are expressed by CD90+ dermal fibroblasts. In SSC skin, PPAR $\alpha$ and PPAR $\gamma$ are expressed by endothelial and CD90+ perivascular cells. In SSC skin, PPAR $\alpha$ and PPAR $\gamma$ appear to be expressed by endothelial cells and $\alpha$-SMA ${ }^{+}$myofibroblasts, but not by $\alpha$-SMA ${ }^{+}$cells localised around blood vessels and postcapillary venules of the superficial plexus. (C) Graphical representation of the expression of PPAR $\alpha^{+}$and PPAR $\gamma^{+}$cells in dermis of normal, ISSc and dSSc skin. All values represent mean \pm SEM; $n=5$ (ISSc), $n=6$ (dSSc) and $n=6$ (normal control). * $p<0.05 ;{ }^{* *} p<0.01$. 


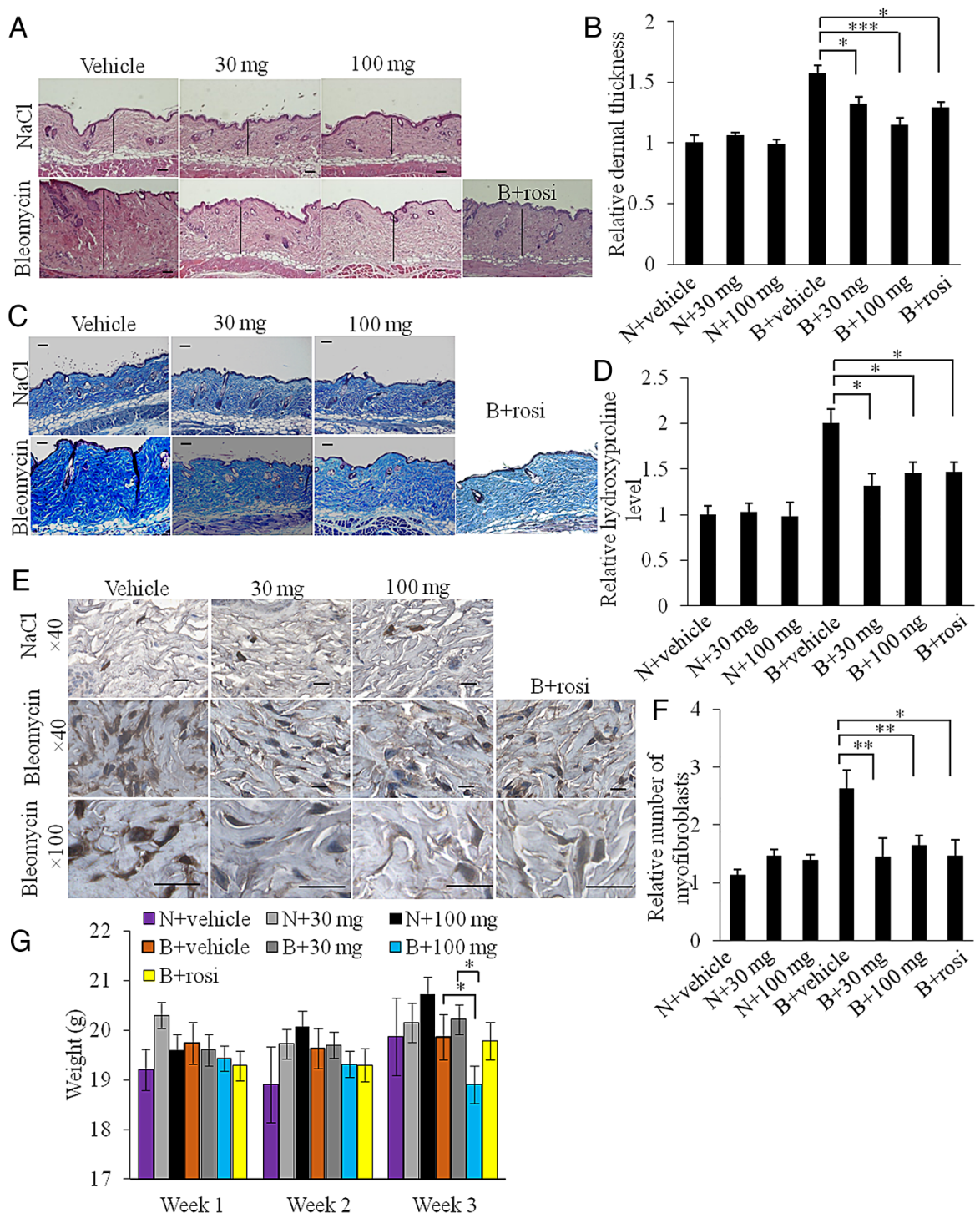

Figure 2 IVA337 attenuates dermal thickness, collagen content and myofibroblast accumulation in preventative model of fibrosis. (A) Representative images of $\mathrm{H} \& \mathrm{E}$-stained sections of mouse skin treated with subcutaneous $\mathrm{NaCl}$ or bleomycin injections. Scale bar is $100 \mu \mathrm{m}$. (B) Graphical representation of dermal thickness of mouse skin harvested after 3 weeks of $\mathrm{NaCl}$ or bleomycin treatment. (N) denotes NaCl; $(\mathrm{B})$ denotes bleomycin; (30 mg) denotes IVA337 at $30 \mathrm{mg} / \mathrm{kg} ;(100 \mathrm{mg})$ denotes IVA337 at $100 \mathrm{mg} / \mathrm{kg}$; (rosi) denotes rosiglitazone at $5 \mathrm{mg} / \mathrm{kg}$. Four high-power field images were captured and two measurements per image were made. Results represent the relative fold change compared with $\mathrm{NaCl}$-treated control mice. (C) Representative images of Masson's trichrome-stained sections of mouse skin harvested after 3 weeks of treatment with NaCl or bleomycin. Scale bar is $100 \mu \mathrm{m}$. (D) Graphical representation of hydroxyproline assay. Results are represented as mean \pm SEM of triplicate measurements obtained from $n \geq 6$ mice (two biopsies per mouse) and shown as relative fold change compared with NaCl-treated control samples. (E) Representative images of $\alpha$-smooth muscle actin (SMA) immunohistochemistry. Negative controls included replacing primary antibodies with normal species-specific lgG. Scale bar is $50 \mu \mathrm{m}$. (F) Graphical representation of relative number of $\alpha$-SMA-positive cells in dermis of $\mathrm{NaCl}$ or bleomycin-treated mice. Results represent the relative fold change compared with $\mathrm{NaCl}$-treated control mice. (G) Body weight changes of mice treated with $\mathrm{NaCl}$ or bleomycin for 3 weeks. All values represent mean $\pm \mathrm{SEM} ; \mathrm{n}=6$ each group. ${ }^{*} \mathrm{p}<0.05 ;{ }^{* *} \mathrm{p}<0.01 ;{ }^{* * *} \mathrm{p}<0.001$.

\section{RESULTS}

\section{PPAR expression is reduced in SSc skin and diminished in bleomycin-treated mouse skin}

We stained limited SSc (ISSc), diffused SSc (dSSc) and normal skin with anti-PPAR $\alpha$, anti-PPAR $\delta$ and anti-PPAR $\gamma$ antibodies. Significant reduction in PPAR $\alpha^{+}$cells was observed in ISSc and dSSc skin compared with normal controls (figure 1A). PPAR $\delta$ was undetectable in SSc and normal skin. SSc skin biopsies were found to have significantly lower number of PPAR $\gamma^{+}$cells than normal skin (figure 1B). Dual immunofluorescence microscopy showed that PPAR $\alpha$ and PPAR $\gamma$ were expressed in dermis of SSc and healthy skin. In healthy skin, PPAR $\alpha$ and PPAR $\gamma$ were expressed by CD90+ (dermal fibroblast and perivascular fibroblastoid cell-type marker ${ }^{24}{ }^{25}$ ) and $\alpha$-smooth muscle actin $(\mathrm{SMA})^{+}$cells, whereas SSc skin showed significant reductions of PPAR $\alpha$ and PPAR $\gamma$ in CD90+ and $\alpha-\mathrm{SMA}^{+}$cells (figure 1A-C).

To test whether bleomycin altered the expression of PPARs in skin, $\mathrm{NaCl}$ and bleomycin-treated mouse skin was immunostained with PPAR $\alpha$, PPAR $\delta$ and PPAR $\gamma$ antibodies. Bleomycin reduced the expression of all three PPAR isoforms, however, levels of PPARs were significantly increased upon treatment with IVA337, suggesting its agonist action in vivo (see online 

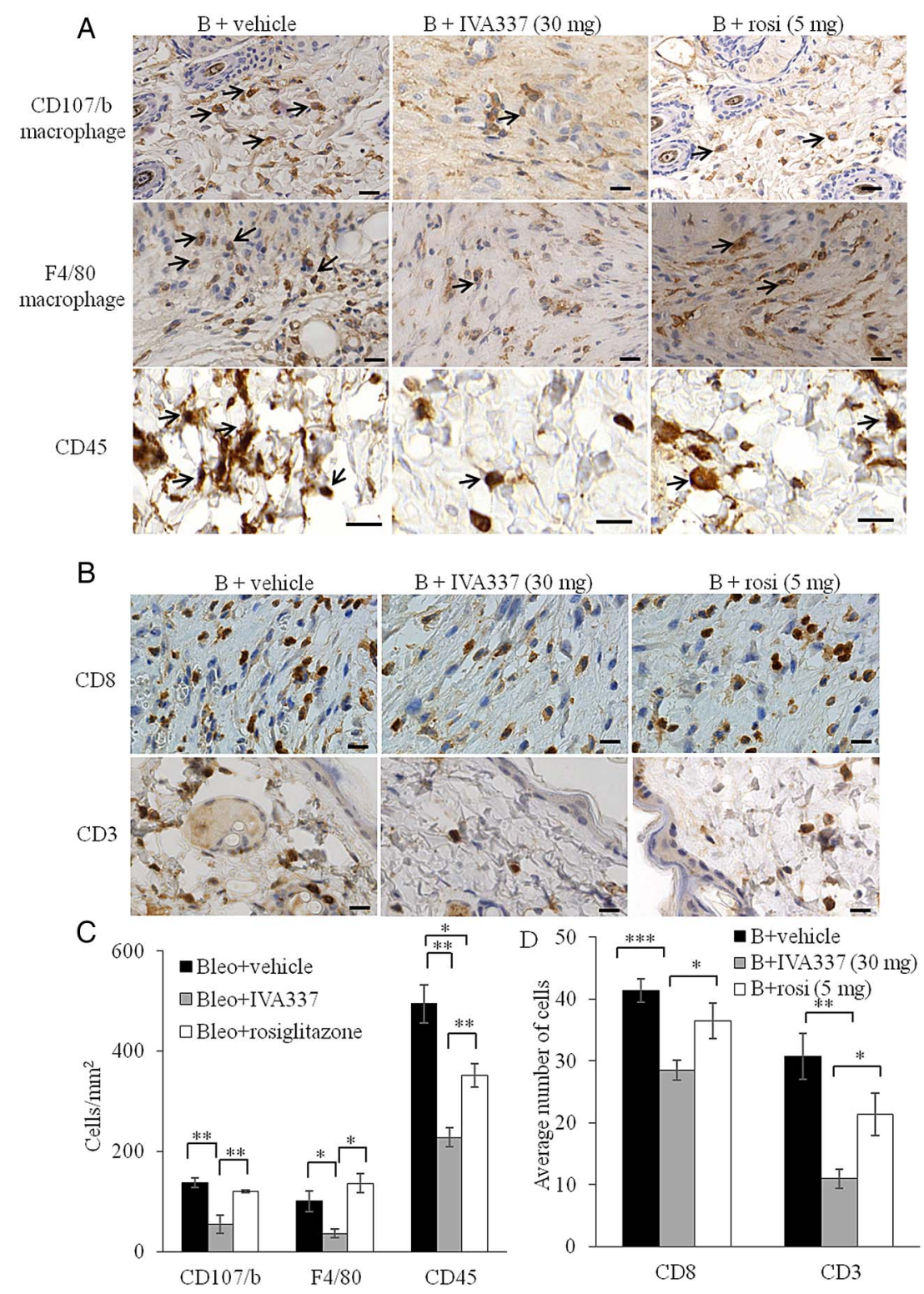

Figure 3 Infiltration of immune effector cells into mouse skin treated with bleomycin and IVA337. (A) Representative immunohistochemistry images of mouse skin incubated with anti-CD107/b (macrophage marker), anti-F4/80 (macrophage marker) and anti-CD45 (leucocyte marker) antibodies. Black arrows indicate immune-positive cells. (B + vehicle) denotes bleomycin/vehicle; (B + IVA337) denotes bleomycin/IVA337 at 30 mg/ $\mathrm{kg}$; (B + rosi) denotes bleomycin/rosiglitazone at $5 \mathrm{mg} / \mathrm{kg}$. Scale bar is $20 \mu \mathrm{m}$. (B) Representative immunohistochemistry images of mouse skin staining for CD8 and CD3 (lymphocytes). (C) Graphical representation of number of CD107/b, F4/40 and CD45+ cells and (D) CD8+ and CD3+ cells in mouse skin treated with either $B+$ vehicle, $B+$ IVA337 at $30 \mathrm{mg} / \mathrm{kg}$ or $B+$ rosi at $5 \mathrm{mg} / \mathrm{kg}$ for 21 days. All values represent mean $\pm S E M ; n=6$ each group. ${ }^{*} \mathrm{p}<0.05 ;{ }^{* *} \mathrm{p}<0.01 ;{ }^{* * *} \mathrm{p}<0.001$.

supplementary figure S1A, B). PPAR $\gamma 1$, but not PPAR $\gamma 2$, activity was increased suggesting that PPAR $\gamma 1$ is preferentially activated in response to IVA337 (see online supplementary figure S4).

\section{IVA337 reduced skin fibrosis in the mouse}

We evaluated the effects of IVA337 using two complementary models of experimental dermal fibrosis. In the first (preventative) approach, skin fibrosis was induced using bleomycin resulting in increased dermal thickness $(\mathrm{p}<0.001 ; \mathrm{NaCl} /$ vehicle vs bleomycin/ vehicle; figure $2 \mathrm{~A}, \mathrm{~B})$, which was attenuated by IVA337 $(\mathrm{p}<0.05$, vehicle vs IVA337 at $30 \mathrm{mg} / \mathrm{kg}$ and $\mathrm{p}<0.001$, vehicle vs IVA337 at $100 \mathrm{mg} / \mathrm{kg}$; figure 2B). Both low and high doses of IVA337 caused a significant decrease of collagenous matrix deposition as evident by weaker Masson's trichrome staining, diminished collagen content as determined by hydroxyproline assay (figure 2C, D) and reduced SHG signal (see online supplementary figure 3A-C).

$\alpha$-SMA labels myofibroblasts that synthesise and deposit extracellular matrix components, making this molecule a reliable marker of fibrosis. ${ }^{6} 26$ After 21 days of bleomycin treatment, a twofold increase in $\alpha$-SMA expression was observed (figure 2E, F). Concurrent bleomycin and IVA337 treatment resulted in significantly reduced number of $\alpha$-SMA-positive myofibroblasts $(\mathrm{p}<0.01$; IVA337 at $30 \mathrm{mg} / \mathrm{kg}$ vs vehicle control; figure $2 \mathrm{~F}$ ). There was no significant change in dermal thickness, hydroxyproline content and myofibroblast count between mice treated with low $(30 \mathrm{mg} / \mathrm{kg})$ and high $(100 \mathrm{mg} / \mathrm{kg})$ doses of IVA337.

Mice were monitored for adverse effects, behaviour pattern and body weight. Clinical signs including body weight, activity, 
A
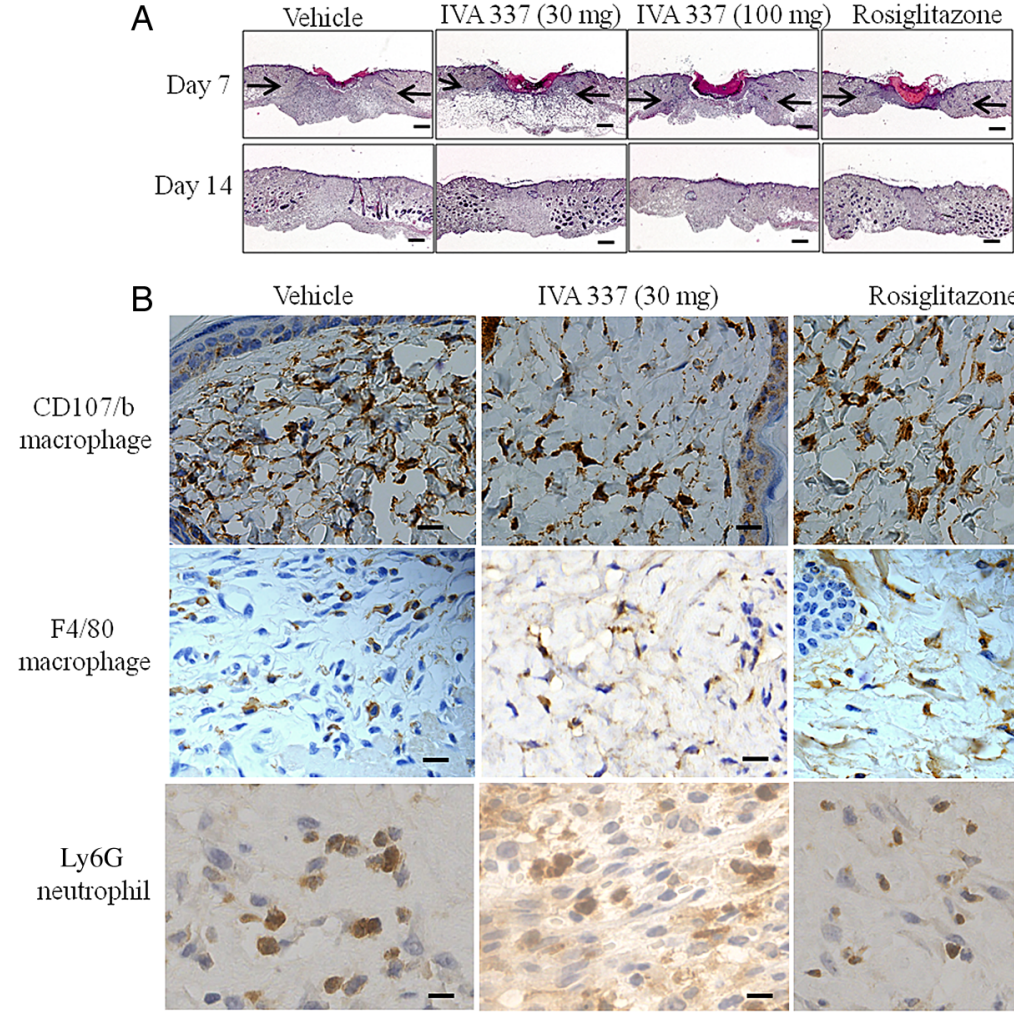

IVA 337 (30 mg)

Rosiglitazone

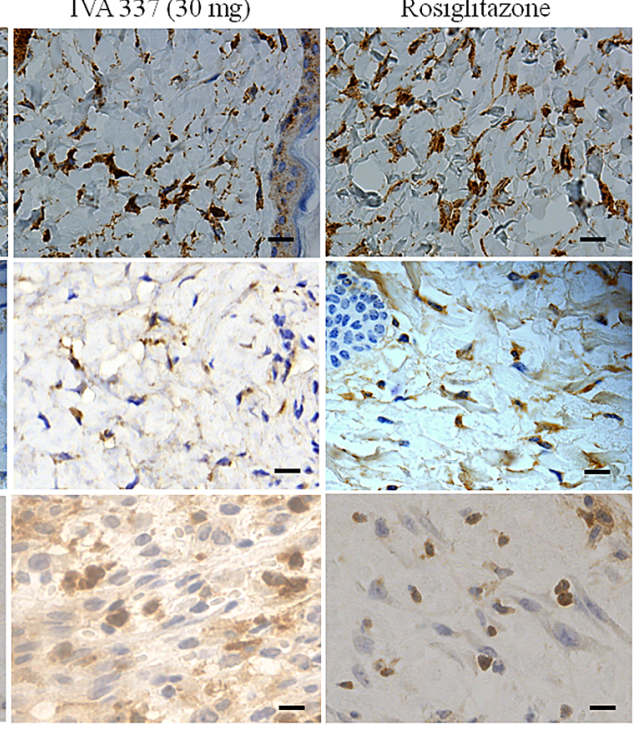

C

B+vehicle

B+IVA337 (30 mg)

$\square \mathrm{B}+\mathrm{IVA} 337(100 \mathrm{mg})$

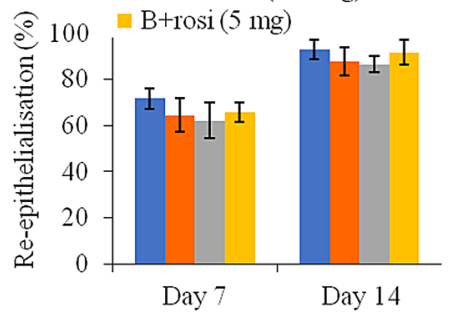

D

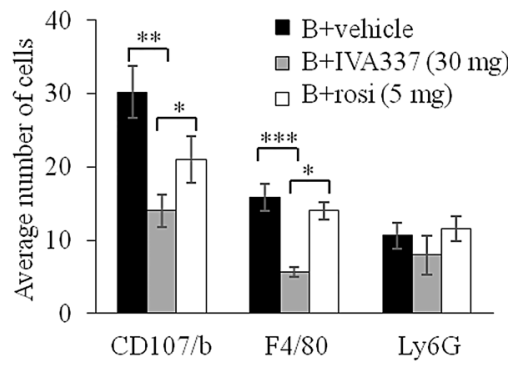

Figure 4 Microscopic and histological analysis of wounds treated with vehicle, IVA337 (30 and $100 \mathrm{mg} / \mathrm{kg}$ ) and rosiglitazone (5 mg/kg). (A) Representative images of H\&E-stained sections of wounds at 7 and 21 days after wounding. Arrows indicate the wound margins. Scale bar is $100 \mu \mathrm{m}$. (B) Representative images of CD107b-stained (macrophage), F4/80-stained (macrophage) and Ly6G-stained (neutrophil) sections of mouse wounds treated with vehicle, IVA337 (30 and $100 \mathrm{mg} / \mathrm{kg}$ ) and rosiglitazone ( $5 \mathrm{mg} / \mathrm{kg}$ ) at 7 days after surgery. Scale bar is $20 \mu \mathrm{m}$. (C) Graphical representation of rate of wound re-epithelialisation at 7 and 14 days after wounding in mice treated with vehicle, IVA337 (30 and $100 \mathrm{mg} / \mathrm{kg}$ ) and rosiglitazone at $5 \mathrm{mg} / \mathrm{kg}$ for 7 and 14 days. (D) Graphical representation of number of CD107b (macrophage), F4/80 (macrophage) and Ly6G (neutrophil) immuno-positive cells in mouse wounds treated with vehicle, IVA337 (30 and $100 \mathrm{mg} / \mathrm{kg}$ ) and rosiglitazone (5 mg/kg) at $7 \mathrm{days}$ after surgery. Scale bar is $20 \mu \mathrm{m}$. All values represent mean \pm SEM; $n=6$ each group. ${ }^{*} p<0.05 ;{ }^{*} p<0.01 ;{ }^{* * *} p<0.001$.

posture, fur texture and skin integrity were recorded daily in the first week of IVA337 administration and then twice a week thereafter until the end point. Although no significant changes were associated with clinical signs such as behaviour, activity, posture, fur and skin texture, small, but significant weight loss was associated with high doses of IVA337. No significant changes in body weight were recorded in mice simultaneously treated with bleomycin and low $(30 \mathrm{mg} / \mathrm{kg})$ doses of IVA337 compared with vehicle controls. Administration of high $(100 \mathrm{mg} / \mathrm{kg})$ doses of IVA337 resulted in reduced body weight compared with vehicle controls $(p<0.05$; bleomycin/IVA337 at $100 \mathrm{mg} / \mathrm{kg}$ vs bleomycin/vehicle; figure $2 \mathrm{G}$ ).

\section{IVA337 reduced inflammation in vivo}

As insights regarding the contribution of PPAR ligands to reduce inflammation become apparent, ${ }^{27} 28$ we hypothesised that activation of all three PPAR isoforms by using IVA337 will modulate acute inflammation in the dermis. After 21 days of concurrent treatment with bleomycin and IVA337, significant reduction in the number of inflammatory cells, as determined by the number of immune-labelled cells (figure 3A-D). Immunohistochemistry studies demonstrated that activation of PPARs with IVA337 induced a significant reduction in the infiltration of macrophages, CD45 + leucocytes and lymphocytes in IVA337-treated mice compared with rosiglitazone-treated counterparts $(\mathrm{p}<0.05$ in $\mathrm{F} 4 / 80, \mathrm{CD} 8$ and CD3; $\mathrm{p}<0.01$ in CD107/b and CD45; figure $3 \mathrm{C}, \mathrm{D})$.

\section{Antifibrotic effects of IVA337 in established fibrosis}

To establish if antifibrotic effects of IVA337 were primarily attributed to its anti-inflammatory properties or whether IVA337 also modulated other fibrotic pathways, treatment with 

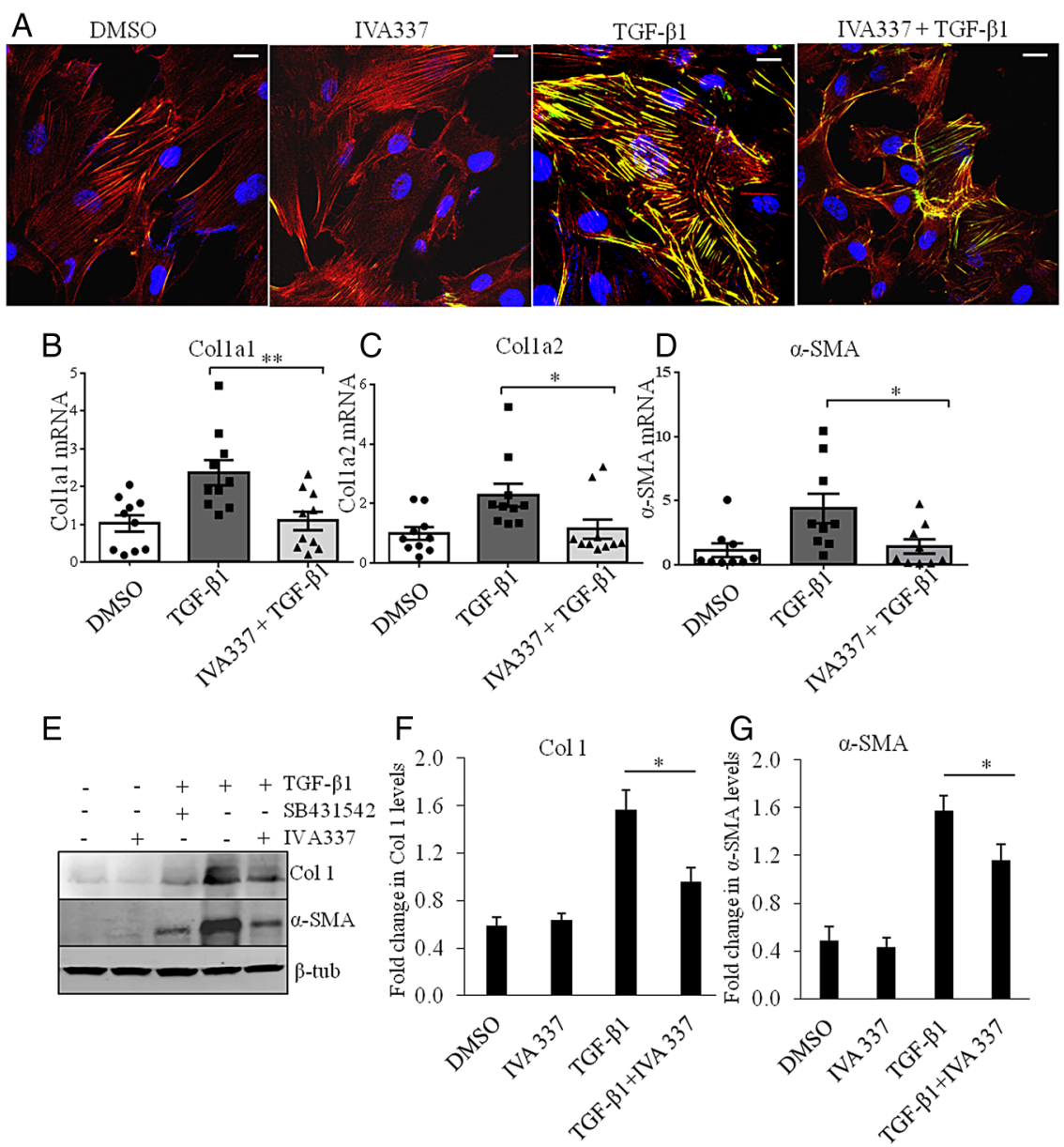

Figure 5 Effect of IVA337 on formation of stress fibres and transforming growth factor (TGF)- $\beta$ signalling in primary human fibroblasts. (A) Systemic sclerosis (SSc) fibroblasts were treated with TGF- $\beta 1(10 \mathrm{ng} / \mathrm{mL})$ and IVA337 $(10 \mu \mathrm{M})$ for $24 \mathrm{~h}$, after which they were fixed with $4 \%$ paraformaldehyde, immunostained for phalloidin (red) and $\alpha$-SMA (green). Scale bar is $25 \mu \mathrm{m}$. (B-D) RNA was isolated from scratch wounded confluent monolayers of SSc fibroblasts. Real-time quantitative (RTq) PCR analysis of (B) Col1A1, (C) Col1A2 and (D) $\alpha$-SMA in response to treatment with IVA337 (10 $\mu$ M) for $24 \mathrm{~h}$. Differences were calculated using the $\mathrm{Ct}$ and comparative $\mathrm{Ct}$ methods for relative quantification. Results were expressed in arbitrary units, where Col1A1, Col1A2 and $\alpha$-SMA genes were normalised with respect to HPRT1gene. All values represent mean $\pm S E M ; n=10$ each group. (E) Representative western blots show the effect of IVA337 (10 $\mu \mathrm{M})$ and the influence of TGF- $\beta 1$ inhibitor SB431542 (10 $\mu \mathrm{M})$ (as a positive control) on TGF- $\beta$-induced expression of collagen 1 and $\alpha$-SMA proteins. ( $F$ and $G$ ) Bar graph based on western blot band densitometry showing the decrease in collagen and $\alpha$-SMA protein expression after incubation with IVA337 $(10 \mu \mathrm{M})$ for $24 \mathrm{~h}(\mathrm{n}=6) .{ }^{*} \mathrm{p}<0.05$; ${ }^{* *} \mathrm{p}<0.01$. $\beta$-tub, $\beta$-tubulin.

IVA337 was initiated after the establishment of dermal fibrosis. In this model of fibrosis regression, dermal thickening and differentiation of fibroblasts into myofibroblasts were significantly reduced in IVA337-treated mice (see online supplementary figure S2A-F). Furthermore, IVA337 was associated with reduced collagen accumulation $(\mathrm{p}<0.01$; IVA337 at $30 \mathrm{mg} / \mathrm{kg}$ vs vehicle control; see online supplementary figure S2D and S3C).

\section{IVA337 does not affect normal wound healing}

Excisional wounds were created on the backs of wild type (WT) mice treated with either IVA337 (30 mg/kg), IVA337 (100 mg/ $\mathrm{kg})$, rosiglitazone $(5 \mathrm{mg} / \mathrm{kg})$ or vehicle for 7 and 14 days. Representative digital images of wounds were captured and planimetric analysis of surface wound area and diameter were determined as described previously ${ }^{18}$ (see online supplementary figure S5A-C). IVA337 (30 mg/kg and $100 \mathrm{mg} / \mathrm{kg}$ ) did not influence the rate of wound closure (figure 4A). Histological analysis of day 7 and 14 wounds showed that the percentage of wound re-epithelialisation, ${ }^{18}$ evaluated by measuring the length of the neoepidermis at day 7 after wounding remained unaffected in IVA337-treated mice compared with vehicle controls (figure 4C). Our studies showed that the expression of PPAR $\delta$, which was undetected in unwounded human skin, is known for its role in epidermal maturation and wound healing, ${ }^{29}$ was significantly higher in day 7 IVA337-treated wounds than in day 14 IVA337 wounds (see online supplementary figure S6A, B).

Given that inflammation is an integral component of normal wound healing and prolonged inflammation may have detrimental effects on wound repair, ${ }^{30} 31$ we next examined the effect of IVA337 on wound inflammation. Day 7 and 14 wounds from mice treated with vehicle, IVA337 (30 and $100 \mathrm{mg} / \mathrm{kg}$ ) and rosiglitazone $(5 \mathrm{mg} / \mathrm{kg}$ ) were stained for CD107b (macrophage), F4/80 (macrophage) and Ly6G (neutrophil) (figure 4B). Although IVA337 decreased the recruitment of macrophages compared with rosiglitazone and vehicle-treated controls, this did not affect the timely closure of IVA337-treated wounds (figure 4D). The number of neutrophils remained unchanged in all groups (figure 4D).

\section{Inhibitory effect of IVA337 on collagen synthesis, $\alpha$-SMA and stress fibre formation}

Fibroblast activation and stress fibre formation are critical in the pathophysiology of fibrosis; ${ }^{26}$ therefore, we hypothesised that antifibrotic effects of IVA337 were, at least partly, due to reduced fibroblast-myofibroblast activation. IVA337 diminished the 


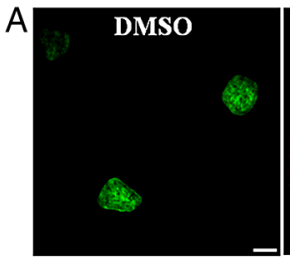

B

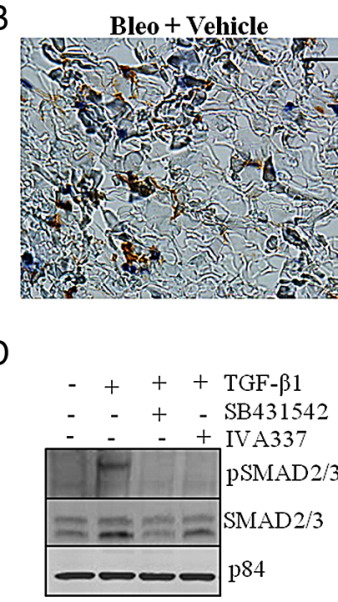

G

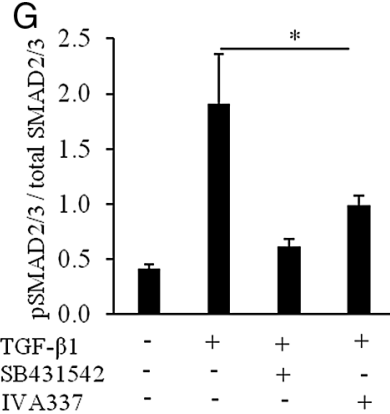

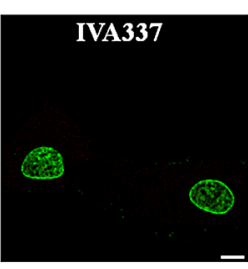

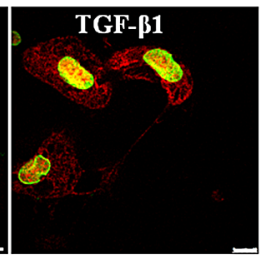

Bleo + IVA337

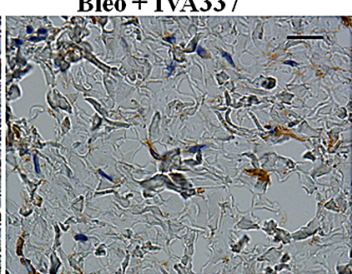

E

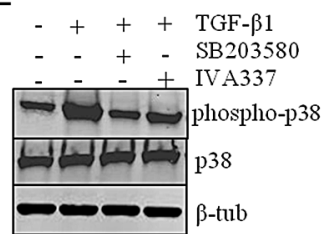

$\mathrm{H}$

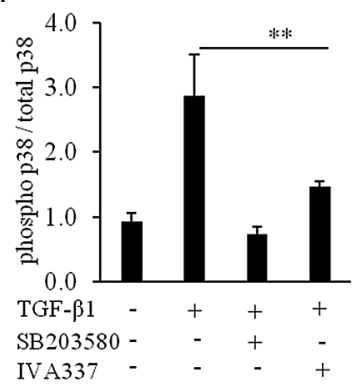

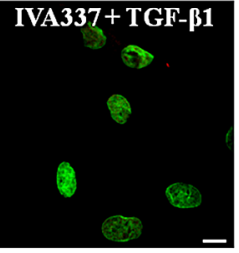

C

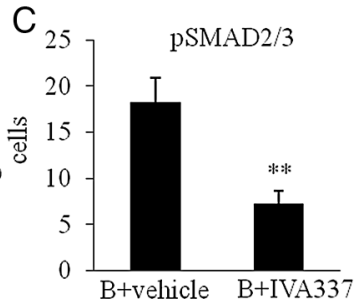

$\mathrm{F}$

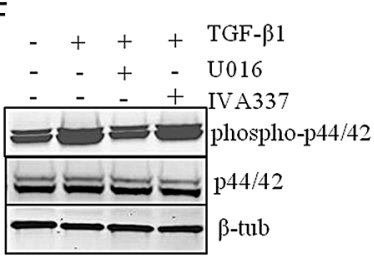

I

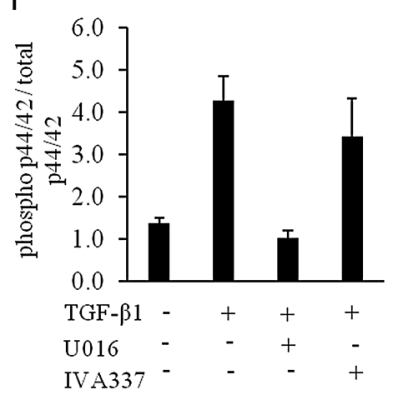

Figure 6 Effect of IVA337 on transforming growth factor (TGF)- $\beta 1$-induced phosphorylation of downstream signalling molecules. (A) Primary systemic sclerosis (SSc) fibroblasts were isolated from fibrotic lesions of four individuals $(n=4)$. Cells were used at passages $2-4$ and incubated in the presence or absence of IVA337 $(10 \mu \mathrm{M})$ for $24 \mathrm{~h}$, fixed with methanol and immunostained for phosphorylated SMAD (pSMAD) $2 / 3$ (red). Nucleus was counterstained with 4',6-diamidino-2-phenylindole (DAPI) (green). TGF- $\beta 1$ (10 ng/mL) caused an increase in pSMAD2/3 (red) immunofluorescence signal, which was found to be present in the nucleus and cytoplasm. Reduction in pSMAD2/3 signal was evident upon incubation with IVA337. Scale bar is $10 \mu \mathrm{m}$. (B) Representative images of mouse skin treated with bleo + vehicle or bleo + IVA337 and stained for pSMAD2/3. (C) Graphical representation of pSMAD2/3 immunohistochemistry of mouse skin treated with bleo + IVA337 or bleo + vehicle for 42 days. All values represent mean $\pm S E M ; n \geq 6$ each group. ${ }^{* *} p<0.01$. (C) Scratch wounded confluent monolayers of SSc fibroblasts were cultured in vitro $(n=4)$ and nuclear extracts were immunoblotted for pSMAD2/3 (nuclear p84 was used as loading control). TGF- $\beta 1(10 \mathrm{ng} / \mathrm{mL}$ ) increased the level of pSMAD2/3 in the nuclear fractions after $1 \mathrm{~h}$ incubation period. (D-G) Preincubation with IVA337 (10 $\mu \mathrm{M})$ and TGF- $\beta 1$ inhibitor SB431542 (10 $\mu \mathrm{M})$ (as a positive control) reduced TGF- $\beta 1$-induced phosphorylation of SMAD2/3. (E and F) Cell extracts from cultured SSc fibroblasts were immunoblotted for (E) phosphorylated p38 (phospho-p38) and total p38 (p38) and (F) phosphorylated p44/42 (phospho-p44/42) and total p44/42 (p44/42). Cytoplasmic $\beta$-tubulin ( $\beta$-tub) was used as loading control. p38 inhibitor SB203580 and U0126, an inhibitor of the mitogen-activated protein kinase/extracellular signal-regulated kinase (ERK) kinase were used as positive control. Treatment with TGF- $\beta 1(10 \mathrm{ng} / \mathrm{mL})$ increased expression of phosphorylated (G) SMAD2/3, (H) p38 and (I) p44/42 after $1 \mathrm{~h}$, whereas IVA337 $(10 \mu \mathrm{M})$ normalised expression of phosphorylated SMAD2/3 and p38, but not phospho-p44/42 in primary dermal fibroblasts derived from patients with SSc. All values represent mean \pm SEM; $n=4$ each group. ${ }^{*} p<0.05 ;{ }^{* *} p<0.01$.

profibrotic effects of TGF- $\beta$ by diminishing differentiation of cultured fibroblasts into myofibroblasts with reduced expression of $\alpha$-SMA and stress fibre formation (figure 5A). To determine if activation of all three PPAR isoforms modified TGF- $\beta$ transcription target genes, we performed gene and protein expression studies. Incubation of SSc fibroblasts with IVA337 significantly reduced the stimulatory effects of TGF- $\beta 1$ on collagen (Col1A1) and $\alpha$-SMA, on both the gene and protein (figure $5 \mathrm{~B}-\mathrm{G}$ ) levels.

\section{Effects of IVA337 on TGF- $\beta$-induced canonical and non-canonical cascades}

In addition to canonical SMAD2/3 pathway, TGF- $\beta$ promotes non-canonical signalling cascades, including p38 mitogen- activated protein kinase (MAPK) and extracellular signalregulated kinase (extracellular signal-regulated kinase (ERK) $1 / 2$ ). ${ }^{32}$ Having demonstrated that IVA337 is a negative regulator of skin fibrosis, we next investigated whether IVA337 affected the translocation of phosphorylated SMAD (pSMAD) 2/3 into the nucleus. Indeed, TGF- $\beta$ caused a robust upregulation of pSMAD2 $/ 3$ in the nucleus and phosphorylation of SMAD2/3 in cultured SSc fibroblasts (figure 6A, D). IVA337 attenuated pSMAD2/3 expression in vitro and in vivo (figure $6 \mathrm{~B}, \mathrm{C}$ ). We speculated that apart from canonical SMAD-dependent pathway, IVA337 may influence non-canonical cascades. The activation of p38 in SSc fibroblasts was analysed after $1 \mathrm{~h}$ of stimulation with TGF- $\beta 1$ and was shown to be inhibited by pretreatment with 
IVA337 or SB203580 (p38 MAPK inhibitor ${ }^{33}$ ), suggesting a relatively direct response (figure $6 \mathrm{E}, \mathrm{H}$ ). SSc fibroblasts showed normal TGF- $\beta$-dependent phosphorylation of p44/42 (ERK1/2) that was reduced by the ERK inhibitor U0126, but not influenced by IVA337 (figure 6F-I).

\section{DISCUSSION}

Our initial finding that expression of PPARs was lower in collagen-rich dermal matrix of SSc skin compared with normal tissue confirmed the importance of this group of nuclear factors in fibrotic skin disorders. This finding was in agreement with studies showing that lack of nuclear factors, including recently described NR4A1, ${ }^{6}$ in fibrotic diseases leads to activation of TGF- $\beta$ signalling and nuclear receptor agonists can overcome this deficiency. ${ }^{9}{ }^{28}$ While PPAR $\alpha$ and PPAR $\gamma$ were detected in skin, PPAR $\delta$ was not expressed. Reportedly low expression of PPAR $\delta$ in normal skin is upregulated in response to wound healing, ${ }^{34}$ which also coincides with acute inflammation. This may explain the presence of PPAR $\delta$ in mouse wounds and its absence in SSc skin, where acute inflammatory is possibly resolved and the dermis is replaced by dense collagen. Our in vivo studies demonstrated that pan PPAR activation reduced fibrogenesis and IVA337 is a negative regulator of dermal fibrosis. Activation of the inflammatory system is associated with the development of fibrosis; ${ }^{4}$ therefore, therapeutic goals in SSc include alleviation of damage caused by early inflammation. PPAR agonists are immunomodulators, ${ }^{11}{ }^{28}$ which partly explains the mechanism of action of their antifibrotic properties. Although overall antifibrotic effects of IVA337 were similar to that of rosiglitazone, IVA337 was more robust in reducing inflammation providing further support for its therapeutic utility in fibrotic diseases. It is conceivable that anti-inflammatory effects of IVA337 could be mediated by activation of PPAR $\gamma$; however, superior anti-inflammatory response associated with administration of IVA337 compared with rosiglitazone alone suggests that pan PPAR agonist IVA337 might target inflammation through a mechanism that increases PPAR $\alpha$ and PPAR $\delta$ activity. In addition to its ability to reduce acute inflammation in a preventative model of fibrosis, IVA337-reduced fibrosis is a clinically relevant model of established skin fibrosis (curative model) via attenuating pSMAD2/3 expression in mouse skin.

Activated PPAR $\alpha$ pathways lead to increased fatty acid oxidation $^{35}$ and energy burning, ${ }^{36}$ which may explain small, but nevertheless significant weight loss in mice treated with high doses of IVA337. Another concern with antifibrotic agents is whether, via their mode of action, they could interfere with the wound equilibrium. Acute inflammation is an integral part of the wound healing, where immune cells such as neutrophils and macrophages $^{30}$ perform important phagocytic functions. ${ }^{31}$ Given the anti-inflammatory effects of IVA337, we used a wound-healing model to investigate the role of IVA337 in wound repair. Although macrophage recruitment was compromised, this did not prevent timely wound closure.

IVA337 attenuated profibrotic effects of TGF- $\beta 1$ by modulating its intracellular effector pSMAD2/3 and reduced the expression of target genes. Although fibrogenesis involves a cross-talk in the system where multiple signals induce more than one cell activity and molecular pathway, our study provided compelling evidence that pan PPAR activation was almost as effective as the TGF- $\beta$ receptor 1 inhibitor SB431542 at concurrently blocking collagen and $\alpha$-SMA expression via dampening canonical TGF- $\beta /$ SMAD2/3 pathway. Provided that deletion of TGF- $\beta$ in mammals interferes with vascular pathology and developmental processes, as well as immune system function, ${ }^{5}$ identification of nuclear receptors, ${ }^{6}$ including PPARs, might provide targets for therapeutic intervention with potentially fewer safety issues than blocking TGF- $\beta$. In keeping with a pathological contribution of some protein kinases to fibrosis, ${ }^{32}$ the finding that IVA337 downregulated TGF- $\beta$-induced p38 MAPK activation, but not ERK1/2, was interesting; however, further studies are warranted to elucidate the effect on downstream and upstream components of these pathways.

Indeed, both anti-inflammatory and antifibrotic properties of IVA337 make it an appealing candidate for further development in fibrotic diseases. PPAR $\gamma$ agonists used for the treatment of metabolic diseases were associated with an increase in the risk of death from cardiovascular causes, ${ }^{14}$ hence, we cannot exclude that simultaneous activation of PPAR $\alpha$, PPAR $\delta$ and PPAR $\gamma$ may exert other biological responses and off-target effects, including vasculogenesis. Hence, further work is required to evaluate the influence of IVA337 on vascular system -important pathological factor in SSc.

\section{CONCLUSION}

Our study furthered the contention that PPAR agonists ameliorate fibrosis and may be amenable to clinical targeting. Two mechanisms were identified to explain the antifibrotic effects of IVA337: (i) dampened immune response and leucocyte infiltration and (ii) inhibition of canonical TGF- $\beta / S M A D$ pathway and non-canonical p38 MAPK signalling. Further preclinical and clinical studies are now warranted to test the efficacy and safety of IVA337 in treating fibrosis associated with SSc.

Acknowledgements We thank M Favier and P Bourdoncle of Institute Cochin, Paris for their excellent expertise.

Contributors NR, CF, SP, JA, TG and MP made substantial contribution to the acquisition and analysis of data. NR drafted the manuscript. NR, JA, YA, J-LJ, PB and J-ML participated in the design of the study, performed the statistical analysis and helped to revise the manuscript. YA and J-LJ conceived and coordinated the project.

Funding This project was funded by INSERM (ATIP/AVENIR) and Inventiva.

Competing interests YA consulted and received research funding from Actelion, Bayer, Biogen Idec, BMS, Genentech/Roche, Inventiva, Medac, Pfizer, Sanofi/ Genzyme, Servier and UCB. YA is a member of the advisory board for the upcoming clinical study of IVA337 in systemic sclerosis. J-ML, PB and J-L are employed by Inventiva.

\section{Patient consent Obtained.}

Ethics approval Hospital Cochin, Paris, France.

Provenance and peer review Not commissioned; externally peer reviewed.

Data sharing statement We have not shared our currently presented data with other members of the scientific community apart from the coauthors whose names appear on the author list.

Open Access This is an Open Access article distributed in accordance with the Creative Commons Attribution Non Commercial (CC BY-NC 4.0) license, which permits others to distribute, remix, adapt, build upon this work non-commercially, and license their derivative works on different terms, provided the original work is properly cited and the use is non-commercial. See: http://creativecommons.org/ licenses/by-nc/4.0/

\section{REFERENCES}

1 Allanore Y, Distler O. Systemic sclerosis in 2014: advances in cohort enrichment shape future of trial design. Nat Rev Rheumatol 2015;11:72-4.

2 Elhai M, Avouac J, Kahan A, et al. Systemic sclerosis: recent insights. Joint Bone Spine 2015;82:148-53.

3 Gabrielli A, Avvedimento EV, Krieg T. Scleroderma. N Engl J Med 2009;360:1989-2003.

4 Ho YY, Lagares D, Tager AM, et al. Fibrosis - a lethal component of systemic sclerosis. Nat Rev Rheumatol 2014;10:390-402.

5 Lafyatis R. Transforming growth factor $\beta$-at the centre of systemic sclerosis. Nat Rev Rheumatol 2014;10:706-19.

6 Palumbo-Zerr K, Zerr P, Distler A, et al. Orphan nuclear receptor NR4A1 regulates transforming growth factor- $\beta$ signaling and fibrosis. Nat Med 2015;21:150-8. 
7 Ghosh AK, Bhattacharyya S, Wei J, et al. Peroxisome proliferator-activated receptor-gamma abrogates Smad-dependent collagen stimulation by targeting the p300 transcriptional coactivator. FASEB J 2009;23:2968-77.

8 Still K, Grabowski P, Mackie I, et al. The peroxisome proliferator activator receptor alpha/delta agonists linoleic acid and bezafibrate upregulate osteoblast differentiation and induce periosteal bone formation in vivo. Calcif Tissue Int 2008;83:285-92.

9 Wei J, Ghosh AK, Sargent JL, et al. PPAR $\gamma$ downregulation by TGF $\beta$ in fibroblast and impaired expression and function in systemic sclerosis: a novel mechanism for progressive fibrogenesis. PLOS ONE 2010;5:e13778.

10 Samah M, El-Aidy Ael R, Tawfik MK, et al. Evaluation of the antifibrotic effect of fenofibrate and rosiglitazone on bleomycin-induced pulmonary fibrosis in rats. Eur $J$ Pharmacol 2012:689:186-93.

11 Galuppo M, Di Paola R, Mazzon E, et al. GW0742, a high affinity PPAR-beta/delta agonist reduces lung inflammation induced by bleomycin instillation in mice. Int $J$ Immunopathol Pharmacol 2010;23:1033-46.

12 Aoki Y, Maeno T, Aoyagi K, et al. Pioglitazone, a peroxisome proliferator-activated receptor gamma ligand, suppresses bleomycin-induced acute lung injury and fibrosis. Respiration 2009;77:311-19.

13 Peters JM, Shah YM, Gonzalez FJ. The role of peroxisome proliferator-activated receptors in carcinogenesis and chemoprevention. Nat Rev Cancer 2012;12:181-95.

14 Nissen SE, Wolski K. Effect of rosiglitazone on the risk of myocardial infarction and death from cardiovascular causes. N Eng/ J Med 2007;356:2457-71.

15 LeRoy EC, Black C, Fleischmajer R, et al. Scleroderma (systemic sclerosis): classification, subsets and pathogenesis. J Rheumatol 1988;15:202-5.

16 Avouac J, Elhai M, Tomcik M, et al. Critical role of the adhesion receptor DNAX accessory molecule-1 (DNAM-1) in the development of inflammation-driven dermal fibrosis in a mouse model of systemic sclerosis. Ann Rheum Dis 2013;72:1089-98.

17 Beyer C, Schett G, Distler 0, et al. Animal models of systemic sclerosis: prospects and limitations. Arthritis Rheum 2010:62:2831-44.

18 Ruzehaji N, Kopecki Z, Melville E, et al. Attenuation of flightless I improves wound healing and enhances angiogenesis in a murine model of type 1 diabetes. Diabetologia 2014;57:402-12

19 Desallais L, Avouac J, Frechet $M$, et al. Targeting IL-6 by both passive or active immunization strategies prevents bleomycin-induced skin fibrosis. Arthritis Res Ther 2014; $16:$ R157

20 Avouac J, Palumbo-Zerr K, Ruzehaji N, et al. The Nuclear Receptor Constitutive Androstane Receptor/NR113 Enhances the Profibrotic Effects of Transforming Growth Factor beta and Contributes to the Development of Experimental Dermal Fibrosis. Arthritis Rheumatol 2014;66:3140-50.
21 Ruzehaji N, Avouac J, Elhai M, et al. Combined effect of genetic background and gender in a mouse model of bleomycin-induced skin fibrosis. Arthritis Res Ther 2015; 17:145.

22 Ruzehaji N, Mills SJ, Melville E, et al. The influence of Flightless I on Toll-like-receptor-mediated inflammation in a murine model of diabetic wound healing. Biomed Res Int 2013;2013:389792.

23 Lei N, Franken L, Ruzehaji N, et al. Flightless, secreted through a late endosome/ lysosome pathway, binds LPS and dampens cytokine secretion. J Cell Sci 2012;125 (Pt 18):4288-96.

24 Lupatov AY, Vdovin AS, Vakhrushev IV, et al. Comparative analysis of the expression of surface markers on fibroblasts and fibroblast-like cells isolated from different human tissues. Bull Exp Biol Med 2015;158:537-43.

25 Pfisterer K, Lipnik KM, Hofer E, et al. CD90+ human dermal stromal cells are potent inducers of FoxP3(+) regulatory T cells. J Invest Dermatol 2015;135:130-41.

26 Huang J, Beyer C, Palumbo-Zerr K, et al. Nintedanib inhibits fibroblast activation and ameliorates fibrosis in preclinical models of systemic sclerosis. Ann Rheum Dis 2016:75:883-90.

27 Mastrofrancesco A, Kovacs D, Sarra M, et al. Preclinical studies of a specific PPARgamma modulator in the control of skin inflammation. J Invest Dermatol 2014;134:1001-11.

28 Wu M, Melichian DS, Chang E, et al. Rosiglitazone abrogates bleomycin-induced scleroderma and blocks profibrotic responses through peroxisome proliferator-activated receptor-gamma. Am J Pathol 2009;174:519-33.

29 Wahli W. Peroxisome proliferator-activated receptors (PPARs): from metabolic contro to epidermal wound healing. Swiss Med Wkly 2002;132:83-91.

30 Martin P. Wound healing-aiming for perfect skin regeneration. Science 1997;276:75-81.

31 Gurtner GC, Werner S, Barrandon Y, et al. Wound repair and regeneration. Nature 2008;453:314-21.

32 Biernacka A, Dobaczewski M, Frangogiannis NG. TGF- $\beta$ signaling in fibrosis. Growth Factors 2011;29:196-202.

33 Jeon $\mathrm{KI}$, Kulkarni $\mathrm{A}$, Woeller CF, et al. Inhibitory effects of PPAR $\gamma$ ligands on TGF- $\beta 1$-induced corneal myofibroblast transformation. Am J Pathol 2014; 184:1429-45.

34 Yessoufou A, Wahli W. Multifaceted roles of peroxisome proliferator-activated receptors (PPARs) at the cellular and whole organism levels. Swiss Med Wkly 2010;140:w13071.

35 Okada-Iwabu M, Yamauchi T, Iwabu M, et al. A small-molecule AdipoR agonist for type 2 diabetes and short life in obesity. Nature 2013;503:493-9.

36 Gao Q, Jia Y, Yang G, et al. PPARalpha-deficient Ob/Ob obese mice become more obese and manifest severe hepatic steatosis due to decreased fatty acid oxidation. Am J Pathol 2015;185:1396-408. 